Nei Santos Duarte 1

Maria Yvone C. Mauro ${ }^{2}$

\section{Análise dos fatores de riscos ocupacionais do trabalho de enfermagem sob a ótica dos enfermeiros}

\author{
Analysis of nursing occupational risk factors from \\ nurses' perspective
}

\begin{abstract}
${ }^{1}$ Fisioterapeuta/Ergonomista, aluna do Programa de Mestrado da Faculdade de Enfermagem, Universidade do Estado do Rio de Janeiro (FENF/UERJ). Rio de Janeiro, RJ.

2 Professora Doutora, Visitante e Titular do Departamento de Enfermagem de Saúde Pública, Faculdade de Enfermagem, Universidade do Estado do Rio de Janeiro (DESP/ FENF/UERJ). Professora Permanente do Programa de Mestrado em Enfermagem, Faculdade de Enfermagem, Universidade do Estado do Rio de Janeiro (FENF/UERJ). Enfermeira do Trabalho / Ergonomista / Pesquisadora do CNPq. Rio de Janeiro, RJ.
\end{abstract}

Contato:

Nei Santos Duarte

Estrada Guandu Sape, 495/101 - Campo Grande - RJ

CEP: 23090-032

E-mail:

nsduartefisio@gmail.com

Recebido: 07/12/2009

Revisado: 22/03/2010

Aprovado: 24/03/2010

\section{Resumo}

Estudo com abordagem quantitativa e descritiva, com objetivo de identificar, na percepção dos enfermeiros chefes de unidades de internação de um hospital universitário, os riscos ocupacionais a que estão expostos. A população foi composta de 30 enfermeiros, chefes de unidade de internação. Na coleta de dados, utilizou-se um questionário estruturado proposto no Guia de Avaliação de Riscos nos Locais de Trabalho de Boix e Vogel (1997), adaptado para a aplicação em estabelecimentos de saúde por Mauro (2001). Os resultados evidenciaram que os fatores de riscos atuam sobre os trabalhadores, propiciando um ambiente desfavorável para a realização das atividades, podendo comprometer a saúde e a vida profissional destes. As variáveis predominantes foram o ambiente e a manutenção preventiva inadequados e os equipamentos de proteção individual e coletiva insuficientes e/ou inadequadamente utilizados pelos profissionais, constrangimentos ergonômicos pela manipulação de carga e postura corporal inadequadas na realização das tarefas, e ritmo de trabalho acelerado pela falta de recursos material e humano. Ressalta-se que a pesquisa proporcionou um diagnóstico referente aos riscos presentes nos ambientes de trabalho da enfermagem, proporcionando um modo operatório eficaz com menor risco de acidentes no processo de trabalho, ambientes menos insalubres e maior satisfação para o profissional e os clientes.

Palavras-chave: risco ocupacional; ergonomia; enfermagem.

\begin{abstract}
The purpose of this study was to identify occupational risks to which head nurses of the hospitalization unit at a university hospital were exposed. It was carried out with a quantitative descriptive approach and involved 30 head nurses. Data was collected through a structured questionnaire proposed by Boix $\&$ Vogel (1997) in their Risk Assessment at the Workplace, which was adapted by Mauro (2001) to be used in hospitals. Results showed that risk factors cause an impact on health professionals and, consequently, provide an unfavorable environment for the development of their professional activities. As a result, it may affect their health and professional life in a negative way. The predominant variables were inappropriate environment and maintenance, insufficient or inadequate use of individual and collective protection equipment, ergonomic constraints on account of inappropriate load and body posture when developing occupational activities, and accelerated work rate due to lack of human and material resources. The research diagnosed nurses' workplace risks, provided information to nurses to perform their tasks in a more effective way, causing fewer accidents during work, less unhealthy environment, and promoting greater satisfaction among nurses and patients.
\end{abstract}

Keywords: occupational risk; ergonomics; nursing. 


\section{Introdução}

Toda avaliação de risco laboral deve ser um momento de reflexão para a otimização da atividade profissional segura, evitando-se, assim, os riscos desnecessários, controlando da melhor forma os riscos que não podem ser eliminados (INSTITUTO NACIONAL DE SEGURIDAD E HIGIENE EN EL TRABAJO, 2008a).

Nesse contexto, incorpora-se a ergonomia, que tem como uma de suas premissas, a análise de serviços, produtos, ferramentas e máquinas, assim como a sua utilização, e os resultados reais que podem ser alcançados, no que concerne às características técnicas dos equipamentos e à análise exaustiva das capacidades e das limitações das pessoas na execução do sistema homem-máquina (MONDELO et al., 1994).

A presença de risco ocupacional no desempenho das atividades laborais do profissional de enfermagem apresenta uma visibilidade multifatorial, devido à diversidade dos fatores de riscos a que estão expostos, dependendo da atividade realizada. Nessa vertente, verifica-se a importância da análise destes riscos para os profissionais.

Marziale e Rodrigues (2002) referem que os trabalhadores de enfermagem, durante a assistência ao paciente, estão expostos a inúmeros riscos ocupacionais causados por fatores químicos, físicos, mecânicos, biológicos, ergonômicos, incluindo os psicossociais, que podem ocasionar doenças ocupacionais e acidentes de trabalho. Castejón (2004) menciona que a avaliação de riscos não é uma atividade estática, e deve ser uma ação dinâmica e contínua, acompanhando as modificações das condições de trabalho.

Almeida e Soares (2002) referem que o projeto de reorganização dos serviços de saúde exige a adoção de uma política comprometida com a melhoria da inserção dos trabalhadores nos serviços de saúde, priorizando os princípios da valorização profissional e da participação dos funcionários, visando produzir mudanças na sua mentalidade e nas suas ações, mediante a qualificação ou a incorporação de novos conhecimentos. Essa proposta visa recuperar uma "postura de respeito e dignidade ao trabalho e aos trabalhadores".

Verifica-se que a qualidade no atendimento hospitalar se relaciona diretamente com as instalações físicas, os equipamentos e os instrumentais utilizados. Cabe aos profissionais de enfermagem, neste contexto, o destaque por serem suas atividades vinculadas diretamente ao "cuidado" durante as 24 horas da assistência prestada, colocando-os em contato permanente com o usuário e os familiares. Decorre daí que o seu trabalho muitas vezes incrementa uma relação de stress, conforme descrito por Oliveira et al. (2008), quando o atendimento à saúde da população envolve muito desgaste físico e emocional dos profissionais.

Brandi (1998), em pesquisa realizada num hospital universitário em 1994, descreve que o ritmo intenso, como consequente sobrecarga de trabalho, pode ser significante na ocorrência do acidente hospitalar com material perfurocortante pelos profissionais de enfermagem. Da mesma forma, verifica-se, na citação de Almeida, Pagliuca e Leite (2005) sobre a importância da conscientização do uso de equipamentos de proteção individual (EPI) pelos profissionais de enfermagem, que o uso correto do EPI colabora essencialmente para a segurança, o bem-estar e a saúde do trabalhador.

Zamberlan e Siqueira (2005) referem que, na atualidade, as organizações encontram-se adotando a terceirização como facilitador do atendimento e da diminuição de custos; cita ainda que a falta de interação intersetorial, por vezes, leva à demora nas informações e à ineficiência na resolução dos problemas apresentados. Os autores citam que a fragmentação do processo de trabalho de enfermagem diante da terceirização dos serviços é inaceitável, porque o atendimento ao ser humano deve ser de maneira única e integral, priorizando, assim, a unicidade e a individualidade de cada um.

A enfermagem, independentemente do trabalho que realiza, deve estar integrada com as demais áreas, na busca de aperfeiçoamento contínuo, para realizar um melhor atendimento, correspondendo à confiança que a clientela dispensa ao cuidador.

O Instituto Nacional de Seguridad e Higiene en el Trabajo (2008b) descreve que os danos ao trabalhador podem ser devidos às condições deficientes de trabalho, como as lesões por acidente de trabalho, as doenças profissionais, a fadiga, a insatisfação, o stress e as patologias inespecíficas.

Diante desse contexto, definiu-se como objeto de estudo, os fatores de risco ambientais do trabalho de enfermagem em estabelecimento hospitalar. Como objetivo, buscou-se identificar, na percepção dos enfermeiros chefes das unidades de internação, os fatores de risco do trabalho de enfermagem em relação ao local, aos equipamentos, às substâncias e aos materiais, fatores ergonômicos e fatores organizacionais.

\section{Materiais e métodos}

O referido estudo é um corte da pesquisa de mestrado intitulada: Fatores de risco no ambiente do trabalho de enfermagem em um hospital universitário do Estado do Rio de Janeiro, apresentada na Faculdade de Enfermagem da Universidade do Estado do Rio de Janeiro (FENF/UERJ), a qual é parte do projeto de pesquisa "Inovação de gestão das condições de trabalho em saúde para hospitais do Sistema Único de Saúde - SUS/ BRASIL”, sob a coordenação da orientadora.

A pesquisa contemplou, em todas as suas etapas, a Resolução nº 196 de 10 de outubro de 1996, sendo submetida e aprovada à análise do Comitê de Ética em Pesquisa do Hospital Universitário, com registro no CEP: 2432. Foram respeitados os direitos dos participantes 
do estudo, e garantido o anonimato quanto à identificação dos participantes da pesquisa.

A coleta de dados ocorreu durante o primeiro semestre do ano de 2009, em um período de oito semanas, e foi utilizado o instrumento desenvolvido a partir do Guia de Avaliação de Riscos nos Locais de Trabalho de Boix e Vogel (1997), adaptado para aplicação em estabelecimentos de saúde por Mauro (2001), composto de 3 Cadernos de Questões.

Para efeito desta pesquisa, foi utilizado apenas o Caderno C, que é composto de um instrumento de observação com questões fechadas, que contemplam as variáveis do estudo sobre o diagnóstico situacional de risco na instituição. Sua formatação contempla um total de 70 alternativas de resposta, classificadas em 5 grupos: locais de trabalho, equipamentos, tecnologia, e instrumentos; substâncias e materiais utilizados; fatores ergonômicos; e fatores organizacionais.

Para o tratamento dos dados, as respostas foram agrupadas e categorizadas para a formação de um banco de dados e para uma posterior análise através do programa de computador: Statistical Package for the Social Sciences (SPSS), versão 15.0.

O tratamento estatístico foi realizado através de uma análise simples e descritiva, com frequências absoluta e relativa.

Ressalta-se que, no período de coleta de dados, uma unidade de internação encontrava-se desativada para obras estruturais, sendo realocada, o que comprometeu as informações de 2 enfermarias. Assim, para evitar o viés na pesquisa pela possível interferência na percepção dos enfermeiros chefes da unidade, definiu-se excluir essas 3 enfermarias da pesquisa, e a coleta foi realizada nas 30 enfermarias restantes.

\section{Análise e discussão dos resultados}

A análise dos riscos tem como objetivos principais a identificação dos riscos, a avaliação do grau desses riscos e a indicação das maneiras de gerenciamento, visando monitorá-los, e, se possível, eliminá-los, conforme descrito por Mauro et al. (2004). Esses autores citam que a visibilidade do acidente de trabalho não deve acontecer apenas no seu contexto, mas em conjunto com os fatores de riscos que o predispõe. Segundo Vogel (1995), a avaliação dos riscos no local de trabalho não deve se limitar apenas à descrição dos riscos ocupacionais a que os profissionais estão expostos.

Ressalta-se a ocorrência de uma limitação na aplicação da metodologia, devido ao curto espaço de tempo para a realização da pesquisa, e à dimensão do campo de estudo, visto que a percepção é uma caracteristica individual e pode sofrer interferência do meio através do pensamento do trabalhador e de sua demonstração nas ações práticas (CORRÊA-FILHO, 1994).
Nesse contexto, constatou-se que os principais resultados pela percepção dos participantes do estudo sobre o setor de trabalho e as instalações (Tabela 1) foram: vestiários e banheiros insuficientes/inadequados (83,3\%); sistemas inadequados de saída de emergência (70\%); sistemas inadequados de prevenção de incêndios ou explosões $(66,7 \%)$; espaço insuficiente para trabalhar em função do excesso de pessoas ou equipamentos; sistema de armazenamento inadequado e/ou inseguro; ventilação/ climatização inadequada dos locais de trabalho; temperatura ambiente inadequada ao tipo de trabalho (60\%).

Quanto a equipamentos, tecnologias e instrumentos utilizados pelos profissionais de enfermagem (Tabela 2), destacaram-se: manutenção preventiva inadequada (80\%); instruções de segurança insuficientes/ inadequadas (70\%); dispositivos de segurança insuficientes/inadequados, utilização inadequada dos equipamentos de proteção individual EPI (60\%).

Em relação às substâncias e aos materiais que são utilizados e/ou manipulados pelos profissionais de enfermagem (Tabela 3), constataram-se: exposição aos riscos biológicos (70\%); desconhecimento sobre os riscos a que estão expostos durante o manuseio dos materiais ou substâncias (63\%); exposição aos riscos químicos em contato com os olhos (40\%); manipulação de substâncias mutagênicas e/ou cancerígenas (33,3\%); exposição aos riscos químicos por inalação (30,0\%); e uso inadequado das etiquetas nos vasilhames $(26,7 \%)$.

Sobre os fatores de riscos ergonômicos (Tabela 4) destacaram-se: distribuição inadequada de pessoal e/ou equipe $(83,3 \%)$; conhecimento insuficiente dos princípios da ergonomia $(73,3 \%)$; enfermarias com ambiente arquitetônico (layout) inadequado dos postos de trabalho (73\%); manipulação de carga inadequada $(63,3 \%)$; falta de ferramentas e/ou instrumentos para a realização das tarefas (60\%); necessidade de adoção de posturas inadequadas do corpo, (53,3\%); espaço inadequado para a realização das atividades (50\%); mobiliário insuficiente nas enfermarias e armazenamento inadequado de materiais (30\%); adoção de posturas corporais por período prolongado $(23,3 \%)$.

Verificou-se que, sobre a organização do trabalho das unidades de internação estudadas (Tabela 5), as principais variáveis foram: falta de recurso material (70\%); pouca oportunidade de promoção (53,3\%); ritmo de trabalho aumentado (40\%); formação contínua insuficiente (33\%); e proteção legal insuficiente em relação aos trabalhadores contratados (30\%).

Nesse sentido, a análise versará sobre as principais variáveis relativas aos fatores de risco ambientais do trabalho de enfermagem, na percepção dos chefes de enfermagem local.

Assim, ressalta-se que os locais de trabalho da equipe de enfermagem evidenciados nesta pesquisa são inadequados ao trabalho dos profissionais, visto que em seu ambiente físico, o espaço, a ventilação e a temperatura não se encontram em conformidade com as Normas Regulamentadoras preconizadas. 
Tabela 1 Respostas positivas às variáveis relativas ao setor de trabalho e instalações, percebidas pelos chefes de enfermagem $(n=30)$ de um hospital universitário, Estado do Rio de Janeiro, julho, 2009

\begin{tabular}{lll}
\hline \multicolumn{1}{c}{ Variável } & \multicolumn{1}{c}{ Chefes de enfermagem } \\
& $n$ & $\%$ \\
\hline Espaço insuficiente para trabalhar por excesso de pessoas ou equipamentos & 12 & 40,0 \\
Desordem e/ou falta de limpeza & 6 & 20,0 \\
Sistema de armazenamento inadequado e/ou inseguro & 18 & 60,0 \\
Falta de segurança nos deslocamentos a pé & 14 & 46,7 \\
Falta de segurança nos deslocamentos mecânicos & 15 & 50,0 \\
Possibilidade de quedas por proteção inadequada de trabalho em altura & 10 & 33,3 \\
Condições inseguras nas instalações elétricas & 15 & 50,0 \\
Condições inseguras nas instalações de gás/pressão & 9 & 30,0 \\
Sistemas inadequados de prevenção de incêndios ou explosões & 20 & 66,7 \\
Sistemas inadequados de saída de emergência & 21 & 70,0 \\
Ventilação/climatização inadequada dos locais de trabalho & 18 & 60,0 \\
Iluminação inadequada ao tipo de trabalho & 10 & 33,3 \\
Temperatura ambiente inadequada ao tipo de trabalho & 18 & 60,0 \\
Ruído ambiental excessivo no trabalho & 85,7 \\
\hline Vestiários e banheiros insuficientes/inadequados & 83,3 \\
\hline
\end{tabular}

Fonte: Pesquisa de campo - HU/2009.

Verificou-se, pela percepção dos participantes da pesquisa, que os ambientes das enfermarias são muito quentes e com iluminação insuficiente para a realização das atividades, o que provoca maior desgaste físico dos profissionais de enfermagem, coerente com Rezende (2003) ao descrever, que, devido ao nível de iluminamento incorreto, os riscos podem predispor os profissionais a doenças, bem como facilitar erros, refletindo nos clientes, comprometendo a qualidade da assistência realizada. Nessa vertente, Dallas (2003) ressalta que o projeto do sistema de ventilação hospitalar precisa de cuidados operacionais de manutenção e de fácil inspeção para prevenir a infecção e favorecer um ambiente confortável para a equipe multiprofissional, o cliente e os seus acompanhantes.

Constatou-se que os enfermeiros participantes citaram que o vestiário e os banheiros são insuficientes para a equipe, o que não condiz com as recomendações da NR 32 (BRASIL, 2005), ocasionando em um constrangimento aos profissionais de enfermagem, visto que os vestiários são, em sua maioria, improvisados e sem nenhuma infraestrutura arquitetônica e de higiene.
Em algumas enfermarias, a improvisação acontece nas varandas, deixando os profissionais expostos às intempéries e aos vetores. Esses dados corroboram com o citado por Vogel (1995) ao referir que os problemas de saúde no trabalho não estão limitados aos efeitos do número de fatores de risco identificados. Segundo o autor, os locais de trabalho não são apenas "lugares" onde existe um número de exposições a riscos especiais, mas são locais de relações que colaboram ou dificultam a conservação da saúde dos trabalhadores.

No quesito sobre incêndios, explosões e sistema de emergência, verificou-se que a maioria das enfermarias possui sistemas inadequados de prevenção de incêndios ou explosões, assim como sistemas inadequados de saída de emergência, corroborando com Hökerberg et al. (2006), que constataram, em pesquisa em unidade hospitalar, a presença dos extintores de incêndio em locais inadequados e mal sinalizados. Citam ainda a inexistência de um programa de treinamento de combate a incêndios, o que, em caso de acidente ou incêndio, pode aumentar a magnitude do evento, visto as condições precárias dos referidos sistemas. 
Ressalta-se que um plano de gerenciamento dos riscos de incêndio é primordial para se compreender o sistema englobado, o processo produtivo, o ambiente físico, econômico, social e político (DUARTE et al., 1998).

Destaca-se que a população percebe a inadequação no sistema de armazenamento de materiais, o que pode gerar sobrecarga física para os profissionais de enfermagem, assim como o risco de acidentes e doenças ocupacionais. Acrescenta-se a isso a existência do risco de contaminação por substâncias manuseadas, em concordância com Veiga (2007). A mesma autora também constatou a falta do planejamento durante a concepção dos estabelecimentos hospitalares, evidenciado nos locais de armazenamento de materiais, favorecendo os acidentes de trabalho na equipe de enfermagem. Corrobora-se o que foi constatado por Hökerberg et al. (2006) sobre os fatores de risco presentes em hospital, decorrentes do armazenamento inapropriado de substâncias químicas, instalações elétricas e de gás, obsoletas, mal sinalizadas e sem manutenção preventiva.

A seguir serão apresentadas as variáveis com maior significância, percebidas pelos profissionais de enfermagem, durante a realização de suas atividades, sobre equipamentos, tecnologias e instrumentos utilizados.

Constatou-se que a manutenção preventiva pela percepção dos participantes da pesquisa encontra-se inadequada, corroborando Aranha e Vieira (2004), que constaram o manuseio incorreto de instrumentos e materiais. Quanto à falta de manutenção, os autores referem que representa desperdícios e citam ainda essa variável no estudo por eles realizado. A ocorrência de instruções de segurança insuficientes/inadequadas e de dispositivos de segurança insuficientes/inadequados contrapõe-se ao enfoque de Dalla (2003), ao referir que a sinalização em ambiente hospitalar deve ser afixada em local visível, facilitando os acessos e a localização do paciente.

Verificou-se que, devido à falta de manutenção dos instrumentos e das ferramentas utilizados pelos profissionais durante a realização de suas atividades, o tempo de vida útil das mesmas leva à degradação do ambiente, o que pode favorecer a elevação da taxa de acidentes de trabalho. Assim, as doenças do trabalho podem surgir pela ocorrência de regulação (improviso) na realização das atividades para suprir a precariedade de instrumentos e ferramentas, ocasionando um aumento da carga cognitiva desses profissionais; no tocante à instituição, ocorrendo um aumento dos gastos na aquisição e/ou no reparo tardio de instrumentais e ferramentas.

Constatou-se que os profissionais de enfermagem utilizam inadequadamente os equipamentos de proteção individual (EPI), o que pode ser ocasionado por falta de conhecimento sobre as consequências do seu desuso ou pela utilização errônea, corroborando o descrito por Paz (2008). Em pesquisa realizadas com profissionais de enfermagem, os trabalhadores relataram a falta de EPI e a sua má utilização, o que, segundo a autora, são fatores que podem contribuir para o aumento do risco biológico no ambiente de trabalho. Esses achados podem ser também pela falta de treinamento ou de desconhecimento da legislação vigente, o que pode expor os profissionais à acidentes de trabalho e à doenças profissionais, estando em não conformidade com a NR 06. Evidenciando o citado por Gir et al. (2000), ao realizar pesquisa em hospital universitário, o descaso pelos profissionais de enfermagem na utilização de medidas de biossegurança indica uma investigação mais profunda desse fato e a necessidade de uma educação continuada, por colocarem em risco a saúde desses trabalhadores.

Tabela 2 Respostas positivas às variáveis relativas a equipamentos, tecnologias e instrumentos, percebidas pelos chefes de enfermagem $(n=30)$ de um hospital universitário, Estado do Rio de Janeiro, julho, 2009

\begin{tabular}{llc}
\hline \multicolumn{1}{c}{ Variável } & \multicolumn{2}{c}{ Chefes de enfermagem } \\
& $n$ & $\%$ \\
\hline Dispositivos de segurança insuficientes/inadequados & 18 & 60,0 \\
Manutenção preventiva inadequada & 24 & 80,0 \\
Instruções de segurança insuficientes/inadequadas & 21 & 70,0 \\
Utilização insegura de máquinas ou ferramentas & 4 & 13,3 \\
Perigo de acidentes por choques ou cortes contra objetos móveis/imóveis & 10 & 33,3 \\
Perigo de acidentes por queimaduras & 2 & 6,7 \\
Perigo de acidentes por descarga elétrica & 7 & 23,3 \\
Proteção inadequada frente ao ruído & 6 & 20,0 \\
Exposição a vibrações por utilização de máquinas ou ferramentas & 2 & 6,7 \\
Fadiga visual por fontes luminosas nos equipamentos de trabalho & 7 & 23,3 \\
Exposição a fontes de calor radiante & 8 & 26,7 \\
Utilização inadequada de equipamentos de proteção individual & 18 & 60,0 \\
\hline
\end{tabular}

Fonte: Pesquisa de campo - HU/2009. 
Através do resultado obtido em relação às substâncias e aos materiais que são utilizados e/ou manipulados pelos profissionais de enfermagem durante a realização de suas atividades, verificou-se que os profissionais de enfermagem não possuem conhecimento sobre os riscos a que estão expostos durante o manuseio de materiais ou de substâncias, apesar de encontrarem-se expostos a riscos químicos por inalação, e pelo contato com os olhos.

Pode-se constatar a ocorrência de manipulação de substâncias mutagênicas e/ou cancerígenas pelos profissionais de enfermagem. Essas substâncias são altamente tóxicas e necessitam de cuidado apurado desde o seu preparo até a infusão no paciente, necessitando de treinamento específico para a sua correta manipulação pelo profissional de enfermagem, não se limitando à transmissão de informações, mas a conscientizá-los, intensivamente, sobre a prevenção de acidentes (RIBEIRO; SHIMIZU, 2007). Esses dados concordam com os achados de Hökerberg et al. (2006). Ao realizarem uma pesquisa sobre a constituição de mapa de risco em uma unidade hospitalar, constataram que os riscos químicos e ergonômicos apontados estavam relacionados principalmente à precária infraestrutura física e ao acondicionamento e manejo inadequados das substâncias químicas.

Soma-se a isso a exposição aos riscos biológicos, os quais já são inerentes à profissão, evidenciando a citação de Muller et al. (2009), de que os profissionais de enfermagem encontram-se expostos ao material biológico por estarem em contato direto com a assistência aos pacientes e devido ao tipo e à frequência de procedimentos realizados sem o real conhecimento do perigo da exposição durante a realização, agravados pelo elevado desconhecimento sobre o correto uso de EPI.
O uso inadequado das etiquetas nos vasilhames, apesar de ser um percentual aparentemente baixo pela percepção dos chefes de enfermagem local, é um agravante para o risco laboral dos profissionais, porque pode ocasionar erros durante o preparo e a administração dos medicamentos.

Os fatores ergonômicos a que estão expostos os profissionais de enfermagem podem ser evidenciados devido ao tempo de existência do Hospital Universitário (59 anos), visto que a maioria das enfermarias estudadas não contemplam vários quesitos referidos na NR 32 e na RDC 50 sobre o seu ambiente arquitetônico (layout), proporcionando um espaço inadequado para a realização das atividades. Isso, corrobora Dalla (2003) ao destacar que a qualidade dos serviços hospitalares realizados encontra-se ligada à funcionalidade das estruturas arquitetônica e organizacional hospitalares. Refere ainda que os serviços de alta tecnologia e a equipe profissional e gerencial são afetados por um espaço arquitetônico não harmônico.

Isso pode acarretar constrangimentos posturais para os profissionais, independentemente das características das tarefas realizadas, pela manipulação de carga inadequada, devido à necessidade de adoção de posturas do corpo inadequadas e algumas vezes por período prolongado para a realização das tarefas. Esse é um fator preponderante para as lesões na coluna vertebral e nos joelhos, dentre outros, estando em concordância com Guedes e Mauro (2001), ao citarem que os problemas musculoesqueléticos estão inseridos entre as principais doenças que acometem os profissionais de enfermagem. Ratificam o citado por Leite, Silva e Merighi (2007) ao relatarem que as queixas relacionadas ao aparelho osteomuscular são uma das maiores causas de dor nos trabalhadores de enfermagem.

Tabela 3 Respostas positivas às variáveis relativas a substâncias e materiais utilizados, percebidas pelos chefes de enfermagem $(n=30)$ de um hospital universitário, Estado do Rio de Janeiro, julho, 2009

\begin{tabular}{llc}
\hline \multicolumn{1}{c}{ Variável } & \multicolumn{2}{c}{ Chefes de enfermagem } \\
& $n$ & $\%$ \\
\hline Utilização de substâncias químicas nocivas e/ou materiais perigosos & 11 & 36,7 \\
Etiquetas inadequadas nos vasilhames & 8 & 26,7 \\
Informação insuficiente sobre os riscos das substâncias ou dos materiais & 11 & 36,7 \\
Falta de segurança nos transportes/armazenamentos de substâncias/materiais & 10 & 33,3 \\
Má qualidade do ar (presença de fumos, gases, vapores, pó e odores) & 6 & 20,0 \\
Riscos químicos por contato com os olhos ou a pele & 12 & 40,0 \\
Riscos químicos por inalação respiratória & 9 & 30,0 \\
Exposição a cancerígenos/mutagênicos & 10 & 33,3 \\
Exposição a produtos alérgicos & 12 & 40,0 \\
Exposição a riscos biológicos & 21 & 70,0 \\
Instalação de proteção coletiva insuficiente ou inadequada & 12 & 40,0 \\
Riscos de acidentes ambientais graves (incêndios, evacuação, explosão) & 7 & 23,3 \\
\hline
\end{tabular}

Fonte: Pesquisa de campo - HU/2009. 
Destaca-se que o mobiliário insuficiente nas enfermarias e o armazenamento inadequado de materiais podem proporcionar um aumento do gasto energético dos profissionais, corroborando com estudos realizados por Guedes (2000) e Lima (2004) sobre a adoção das posturas dos profissionais de enfermagem. Nesse sentido, Paz (2008) ressalta a importância dos investimentos financeiros para a melhora das condições de trabalho da enfermagem, como alterações nos postos de trabalho, aquisição de materiais, equipamentos e mobiliários. A autora refere ainda que o investimento empregado proporciona retorno financeiro, pela redução dos gastos com a saúde do trabalhador, prevenindo acidentes e absenteísmos.

Verificou-se que, além do exposto, as equipes não possuem número suficiente de profissionais para a plena realização das tarefas, ocorrendo má distribuição das equipes e, em função disso, corroborando o descrito por Lima (2004), segundo o qual os auxiliares de enfermagem são levados a realizar tarefas designadas a outras categorias profissionais, aumentando sua demanda física, cognitiva e psicossocial.

A falta de ferramentas e/ou instrumentos para a realização das tarefas ocasionam, nos profissionais, uma sobrecarga ocupacional que é agravada pelo fato de os profissionais de enfermagem, em sua grande maioria, não possuírem conhecimento suficiente dos princípios da ergonomia (que, além da conscientização corporal, engloba conhecimento cognitivo e sobre o ambiente físico), corroborando o constatado por Ribeiro e Shimizu (2007), cujos dados alcançados, ao realizarem pesquisa em um hospital universitário no Distrito Federal, mostraram um preparo deficiente e uma falta de treinamento e de capacitação de profissionais, agregados a ambientes físicos inadequados e à falta de material apropriado.

A organização do trabalho nas instituições, independentemente da esfera administrativa a que estão vinculadas (pública ou privada), é um fator determinante para a realização das atividades pelos profissionais e a sua produtividade.

No caso do estabelecimento de saúde, um atendimento de excelência para os clientes em um ambiente digno para os profissionais que nele atuem, ficou explícito através das variáveis percebidas pelos enfermeiros chefes de unidade. Paz (2008) refere, em pesquisa realizada, que, dentre os fatores de riscos do trabalho de enfermagem, os ocupacionais relacionados com a organização do trabalho, obtiveram maiores repercussões para a saúde do trabalhador, corroborando os dados apresentados nesta pesquisa, segundo a qual a organização do trabalho encontra-se insatisfatória no Hospital Universitário, apesar de aparentemente ser um percentual baixo.

Tabela 4 Respostas positivas às variáveis relativas aos fatores ergonômicos, percebidas pelos chefes de enfermagem $(n=30)$ de um hospital universitário, Estado do Rio de Janeiro, julho, 2009

\begin{tabular}{lll}
\hline \multicolumn{1}{c}{ Variável } & \multicolumn{2}{c}{ Chefes de enfermagem } \\
& $n$ & $\%$ \\
\hline Desenho arquitetônico inadequado dos postos de trabalho em geral & 22 & 73,3 \\
Espaço reduzido de trabalho para a tarefa & 15 & 50,0 \\
Distribuição inadequada de pessoal e/ou equipe & 25 & 83,3 \\
Ferramentas insuficientes ou inadequadas & 18 & 60,0 \\
Cadeiras e assentos insuficientes/inadequados & 22 & 73,3 \\
Manutenção excessiva de uma mesma postura no trabalho & 10 & 33,3 \\
Necessidade de adotar posturas forçadas não confortáveis & 16 & 53,3 \\
As tarefas não permitem mudanças frequentes de postura & 6 & 20,0 \\
Repetitividade excessiva de movimentos & 10 & 33,3 \\
Manejo inadequado de cargas (peso, volume, altura, deslocamentos) & 19 & 63,3 \\
Manejo prolongado de cargas sem pausas suficientes & 7 & 23,3 \\
Armazenamento inadequado de cargas que impede a correta manipulação & 9 & 30,0 \\
Contaminação externa (resíduos, emissões de calor, vetores, outros) & 6 & 22,0 \\
Conhecimento ergonômico insuficiente/inadequado do trabalhador & 22 & 73,3 \\
\hline
\end{tabular}

Fonte: Pesquisa de campo - HU/2009. 
Esse fator é um somatório de vários outros fatores, dentre eles a pouca oportunidade de promoção, apesar da instituição possuir plano de cargos e salário. Esse fato corrobora com o descrito por Boix e Vogel (2003) ao ressaltarem que a importância do trabalho no processo saúde-doença, através das relações sociais, são elementos-chave na construção cultural da saúde. Referem ainda que a base social, a solidariedade, a satisfação no ambiente do trabalho ou a autonomia pessoal estão diretamente relacionados com a saúde no trabalho.

Stumm et al. (2006) ressaltam, em sua pesquisa, a importância do enfermeiro coordenador de um centro cirúrgico e do relacionamento interpessoal, destacando que o mesmo deve ser vigilante às características individuais dos diferentes profissionais para administrar sua equipe e a equipe médica. Citam ainda que a relação interpessoal problemática entre as equipes repercute no processo de trabalho e pode ocasionar danos à saúde dos profissionais, o que evidencia o achado desta pesquisa.

Pode-se verificar que o relacionamento interpessoal dos profissionais é de forma inter e multidisciplinar, e a proteção legal é insuficiente em relação aos trabalhadores contratados, corroborando o descrito por Tavares (2006) ao descrever que o trabalho com o público é uma tarefa penosa e que as empresas devem realizar treinamento de relações interpessoais. Essas variadas formas de relacionamento também colaboram para a insatisfa- ção dos profissionais de enfermagem, sendo esse fator determinante da motivação dos trabalhadores, podendo ocasionar problemas psicossociais.

Vale ressaltar o descrito por Sznelwar et al. (2004), segundo o qual as demandas referentes aos trabalhadores, à logística, à organização do trabalho, assim como à infraestrutura, mostram as grandes dificuldades para o bom funcionamento do sistema hospitalar, devido à complexidade do sistema.

Nesse sentido, verificou-se que a organização do trabalho das unidades de internação estudadas encontra-se comprometida com a realização das atividades pelos profissionais com ritmo de trabalho aumentado e com a falta de recurso material, corroborando Stumm et al. (2006) ao ressaltarem que a falta de materiais e equipamentos básicos demonstra como verdadeira a situação do setor de saúde no Brasil, gerando insatisfação à equipe. Esse fato é descrito por Lima (2004) ao evidenciar que algumas atividades se sobrepõem devido ao curto espaço de tempo para a sua realização, à falta de material e medicamentos e aos equipamentos inadequados, dificultando o seu desenvolvimento. Ressalta-se que a equipe de enfermagem geralmente atua em instituições hospitalares com déficits de recursos humanos e materiais, proporcionando um trabalho mais penoso e provocando agravos à saúde do trabalhador (LEITE; SILVA; MERIGHI, 2007).

Tabela 5 Respostas positivas às variáveis relativas aos fatores organizacionais do trabalho, percebidas pelos chefes de enfermagem $(n=30)$ de um hospital universitário, Estado do Rio de Janeiro, julho, 2009

\begin{tabular}{|c|c|c|}
\hline \multirow{2}{*}{ Variável } & \multicolumn{2}{|c|}{ Chefes de enfermagem } \\
\hline & $n$ & $\%$ \\
\hline Organização do trabalho insatisfatória & 11 & 36,7 \\
\hline Tarefas aborrecidas ou monótonas & 3 & 10,0 \\
\hline Ritmo de trabalho/pressão de tempo excessivo & 12 & 40,0 \\
\hline Recursos insuficientes para alcançar os objetivos ou prazos fixados & 21 & 70,0 \\
\hline Trabalho inadequado em equipe ou sem colaboração & 4 & 13,3 \\
\hline Falta de autonomia para os trabalhadores & 4 & 13,3 \\
\hline Duração da jornada e/ou organização de horários e turnos inadequados & 1 & 3,3 \\
\hline Dificuldades em compatibilizar trabalho e vida social/familiar & 3 & 10,0 \\
\hline Canais de participação e de consulta com a chefia insuficientes ou inadequados & 0 & 0,0 \\
\hline Poucas oportunidades de formação contínua & 10 & 33,3 \\
\hline Poucas oportunidades de promoção no trabalho & 16 & 53,3 \\
\hline Relações insatisfatórias com os dirigentes ou encarregados & 2 & 6,7 \\
\hline Relações insatisfatórias entre os trabalhadores & 3 & 10,0 \\
\hline Relações insatisfatórias com os clientes/usuários & 1 & 3,3 \\
\hline Divisão de trabalho em tarefas "de mulheres" e tarefas "de homens" & 1 & 3,3 \\
\hline Maior exposição ao risco dos trabalhadores não efetivos & 4 & 13,3 \\
\hline Proteção legal insuficiente aos trabalhadores & 9 & 30,0 \\
\hline
\end{tabular}

Fonte: Pesquisa de campo - HU/2009. 
Rezende (2003) apontou a importância do Programa de Educação Permanente de alta qualidade, visando proporcionar, aos profissionais de enfermagem, novos conhecimentos para a execução com excelência de suas tarefas laborais, o que se encontra em concordância com a percepção dos enfermeiros participantes desta pesquisa. Eles apontaram como insuficiente a formação contínua, indicando que ela seja complementar ao conhecimento dos profissionais, de acordo com as necessidades (técnicas) laborais desenvolvidas, haja vista as particularidades das atividades desenvolvidas de acordo com a enfermaria em que estejam desempenhando, pois, compreendendo os processos de produção existentes e adequando as tarefas aos trabalhadores, ocorrerá uma contribuição para minimizar ou mesmo dirimir o problema dos riscos à saúde no ambiente hospitalar (SZNELWAR et al., 2004).

Os achados desta pesquisa corroboram a literatura pesquisada no que concerne aos fatores de riscos do trabalho de enfermagem em hospitais universitários e em outras instituições de saúde. Destaca-se que, devido à estrutura complexa que constitui os hospitais universitários, é importante que se maximize o controle do processo de gestão hospitalar, otimizando os serviços. Isso pode ser conseguido revisando-se as estratégias para o planejamento das ações de saúde e através de uma análise profunda das condições de trabalho, utilizando a percepção dos profissionais de saúde sobre os problemas encontrados no contexto do ambiente de trabalho (CIAMPONE et al., 1998; SILVA; MARZIALE, 2003; DALLORA; FORSTER, 2008; BAULI; MATSUDA, 2009).

\section{Conclusão}

Os resultados encontrados através da percepção dos enfermeiros chefes de unidade de internação sobre os fatores de riscos do trabalho de enfermagem evidenciam a importância dos fatores ambientais e ergonômicos, os quais, de forma direta ou indireta, proporcionam aos profissionais um ambiente desfavorável para a realização das atividades e podem comprometer a sua vida profissional devido à extrema solicitação cognitiva e física desses trabalhadores.

Destaca-se a importância dos impactos econômicos e laborais ocasionados à instituição e aos profissionais de enfermagem, principalmente como causadores de absenteísmo e de sequelas de lesões, com que são acometidos devido aos acidentes de trabalho.

Verifica-se que os fatores de riscos ambientais no trabalho de enfermagem encontram-se presentes diariamente nas suas atividades, cabendo aos gestores fazer uma avaliação dos mesmos de forma a minimizarem os seus impactos sobre a vida desses profissionais.

É recomendável proporcionar um modo operatório eficaz com menor risco de acidentes para os próprios profissionais e clientes através do gerenciamento de risco eficiente, visando a ambientes menos insalubres e com maior satisfação na profissão, o que, para a instituição, significa um sistema mais produtivo com visibilidade favorável, seja para os gestores, os clientes, ou para a mídia, facilitando a captação de recursos materiais e humanos para as necessidades reais da organização.

Ressalta-se que, independentemente da limitação metodológica apresentada, a pesquisa proporcionou um diagnóstico para os gestores da instituição concernente aos riscos ambientais presentes nos locais de trabalho. É importante desencadear um processo de treinamento eficaz e uma avaliação do impacto, junto aos trabalhadores, sobre os problemas levantados e a possibilidade de intervenção nos mesmos através de um planejamento eficiente.

A metodologia aplicada pode contribuir, de maneira efetiva, no processo de reconhecimento dos riscos ocupacionais a que se encontram expostos os trabalhadores de enfermagem. Sugere-se reaplicar a metodologia em outras pesquisas para proporcionar o aprimoramento e a funcionalidade na gestão de riscos ocupacionais, assim como a sua aplicabilidade em outras categorias profissionais.

\section{Referências}

ALMEIDA, A. H.; SOARES, C. B. A dimensão política do processo de formação de pessoal auxiliar: a enfermagem rumo ao SUS. Revista Latino-Americana de Enfermagem, Ribeirão Preto, v. 10, n. 5, p. 629-636, set./out. 2002.

ALMEIDA, C. B.; PAGLIUCA, L. M. F.; LEITE, A. L. A. S. Acidentes de trabalho envolvendo os olhos: avaliação de riscos ocupacionais com trabalhadores de enfermagem. Revista Latino-Americana de Enfermagem, Ribeirão Preto, v. 13, n. 5, p. 708-716, set./out. 2005.

ARANHA, G. T. C.; VIEIRA, R. W. Estudo de um dos indicadores do custo da qualidade: o desperdício. Revista de Administração em Saúde, São Paulo, v. 6, n. 23, abr./ jun. 2004. Disponível em: <http://www.cqh.org.br/files/ ras23_Guiomar02.pdf>. Acesso em: 13 ago. 2009.

BAULI, J. D.; MATSUDA, L. M. Diagnóstico situacional do serviço de enfermagem de hospital de ensino sob a ótica dos profissionais de nível médio. Revista de Administração em Saúde, São Paulo, v. 11, n. 43, abr./jun. 2009. Disponível em: < http://www.cqh.org.br/ files/RAS43\%20-\%201-\%20\%20Diagn\%C3\%B3stico. pdf $>$. Acesso em: 13 ago. 2009.

BOIX, P.; VOGEL, L. La evolución de riesgos en los lugares de trabajo: guía para una intervención sindical. Bruxelas: BTS, 1997. 
. Participacion de los trabajadores. Disponível em: <www.srt.gov.ar/super/eventos/2003/ Participacion_Boix_Vogel.pdf $>$. Acesso em: 29 ago. 2009 .

BRANDI, S. Ocorrência de acidentes do trabalho por material pérfuro-cortante entre trabalhadores de um hospital universitário da cidade de Campinas (SP). Estado de São Paulo, Revista da Escola de Enfermagem da USP, São Paulo, v. 32, n. 2, p. 124-133, 1998.

BRASIL. Ministério do Trabalho e Emprego. NR 32 Segurança e Saúde no Trabalho em Serviços de Saúde. Portaria GM nº 485, de 11 de novembro de 2005. Diário Oficial da União, Brasília, DF, 16 nov. 2005. Disponível em: <http://www.mte.gov.br/legislacao/ normas_regulamentadoras/nr_32.pdf $>$. Acesso em: 13 abr. 2008.

CASTEJÓN, E. Evaluación de riesgos. In: BENAVIDES, F. G.; RUIZ-FREITOS, C.; GARCÍA, A. G. Salud laboral: conceptos y técnicas para la prevención de riesgos laborales. 2. ed. Barcelona: Masson, 2004. p. 223-234.

CIAMPONE, M. H. T. et al. Processo de planejamento na prática da enfermagem em um hospital de ensino. Revista da Escola de Enfermagem da USP, São Paulo, v. 32, n. 3, p. 273-280, out. 1998.

CORREAA-FILHO, H. R. Percepção de riscos na ocupação precedendo lesões do trabalho: um estudo no Município de Campinas, São Paulo, 1992-1993. 1994. 197 f. Tese (Doutorado)-Faculdade de Saúde Pública, Universidade de São Paulo, São Paulo, 1994.

DALLA, T. C. M. Estudo da qualidade do ambiente hospitalar como contribuição na recuperação de pacientes. 2003. 169 f. Dissertação (Mestrado em Engenharia Civil)-Universidade Federal do Espírito Santo, Espírito Santo, 2003.

DALLORA, M. E. L. V.; FORSTER, A. C. A importância da gestão de custos em hospitais de ensino considerações teóricas. Medicina, Ribeirão Preto, v. 41, n. 2, p. 135-142, abr./jun. 2008. Disponível em:<http:// www.fmrp.usp.br/revista $>$. Acesso em: 10 set. 2009.

DUARTE, D.; LEITE, M. S.; PONTES, R.

Gerenciamento dos riscos de incêndios. In: ENCONTRO NACIONAL DE ENGENHARIA DE PRODUÇÃO, 18, 1998. Anais eletrônicos... Brasil: Abepro, 1998. Disponível em: <http://www.abepro. org.br/biblioteca/ENEGEP1998_ART366.pdf $>$. Acesso em: 22 nov. 2009.

GIR, E. et al. Alterações na prática profissional de enfermeiros de um hospital de ensino do interior paulista, em conseqüência ao surgimento do HIV/ AIDS. Revista Gaúcha de Enfermagem, Porto Alegre, v. 21, n. 2, p. 37-54, 2000.

GUEDES, E. M. Distúrbios osteomusculares e o trabalho de enfermagem hospitalar: estudo com os auxiliares de enfermagem em unidade de ortopedia. 2000. 160 f. Dissertação (Mestrado em Enfermagem)-
Faculdade de Enfermagem, Universidade do Estado do Rio de Janeiro, Rio de Janeiro, 2000.

GUEDES, E. M.; MAURO, M. Y. C. (Re)visando os fatores de risco e as condições de trabalho da enfermagem hospitalar. Revista Enfermagen Uerj, Rio de Janeiro, v. 9, n. 2, p. 144-151. 2001.

HOKERBERG, Y. H. M. et al. O processo de construção de mapas de risco em um hospital público. Ciência $\mathcal{E}$ Saúde Coletiva, Rio de Janeiro, v. 11, n. 2, p. 503-513, 2006.

INSTITUTO NACIONAL DE SEGURIDAD E HIGIENE EN EL TRABAJO - INSHT. Evaluación de las condiciones de trabajo en la PYME. 5. ed. España: INSHT, 2008a. Disponível em: < http://www.insht. es/portal/site/Insht>. Acesso em: 31 jul. 2008.

. Manual de procedimientos de prevención de riesgos laborales. Guía de elaboración - INSHT, 2008b. Disponível em: < http://www.mtas.es /INSHT/ PRACTICE/MAN_PROC.HTM > . Acesso em: 31 jul. 2008.

LEITE, P. C.; SILVA, A.; MERIGHI, M. A. B. A mulher trabalhadora de enfermagem e os distúrbios osteomusculares relacionados ao trabalho. Revista $d a$ Escola de Enfermagem da USP, São Paulo, v. 41, n. 2, p. 287-291, 2007.

LIMA, M. C. T. F. C. Análise das condições ergonômicas da situação de trabalho dos auxiliares de enfermagem em uma unidade de internação hospitalar. 2004. $93 \mathrm{f}$. Dissertação (Mestrado Profissional em Engenharia)Escola de Engenharia, Universidade Federal do Rio Grande do Sul, Porto Alegre, 2004.

MARZIALE, M. H. P.; RODRIGUES, C. M. A produção científica sobre os acidentes de trabalho com material perfurocortante entre trabalhadores de enfermagem. Revista Latino-Americana de Enfermagem, Ribeirão Preto, v. 10, n. 4, p. 571-577, jul./ago. 2002.

MAURO, M. Y. C. A situação de saúde dos trabalhadores de transporte de cargas de uma companhia em Volta Redonda-RJ, Brasil. In: SEMANA DE INICIAÇÃO CIENTÍFICA/UERJ, 10, 2001, Rio de Janeiro. Anais... Rio de Janeiro: UERJ, 2001. 1 CD. v. 1, p. 189.

MAURO, M. Y. C. et al. Riscos ocupacionais em saúde. Revista Enfermagem UERJ, Rio de Janeiro, v. 12, p. 338-345, 2004.

MONDELO, P.; GREGORI, E.; BARRAU, P. Ergonomía 1: fundamentos. Barcelona: Universitat Politècnica de Catalunya-UPC; SL Jordi Girona Salgado, 1994.

MULLER, L. R. et al. Riscos ocupacionais dos trabalhadores de enfermagem: uma revisão bibliográfica. Disponível em: <www.abennacional. org.br/2SITEn/Arquivos/N.111.pdf> . Acesso em: 04 nov. 2009.

OLIVEIRA, D. C. et al. A política pública de saúde brasileira: representação e memória social de 
profissionais. Cadernos de Saúde Pública, Rio de Janeiro, v. 24, n. 1, p. 197-206, jan. 2008.

PAZ, A. F. Relação entre fatores de risco no ambiente hospitalar e a saúde dos trabalhadores de enfermagem. 2008. 105 f. Dissertação (Mestrado em Enfermagem)Faculdade de Enfermagem, Universidade do Estado do Rio de Janeiro, Rio de Janeiro, 2008.

REZENDE, M. P. Agravos à saúde de auxiliares de enfermagem resultantes da exposição ocupacional aos Riscos Físicos. 2003. 114 f. Dissertação (Mestrado)Escola de Enfermagem de Ribeirão Preto, Universidade de São Paulo, Ribeirão Preto, 2003.

RIBEIRO, E. J. G.; SHIMIZU, H. E. Acidentes de trabalho com trabalhadores de enfermagem. Revista Brasileira de Enfermagem, Rio de Janeiro, v. 60, n. 5, p. 535-540, set./out. 2007.

SILVA, D. M. P. P.; MARZIALE, M. H. P. Problemas de saúde responsáveis pelo absenteísmo de trabalhadores de enfermagem de um hospital universitário. Acta Scientiarum, Health Sciences, Maringá, v. 25, n. 2, p. 191-197, 2003.

STUMM, E. M. F.; MAÇALAI, R. T.; KIRCHNER R. M. Dificuldades enfrentadas por enfermeiros em um centro cirúrgico. Texto e Contexto-Enfermagem, Florianópolis, v. 15, n. 3, p. 464-471, jul./set. 2006.

SZNELWAR, L. I. et al. Análise do trabalho e serviço de limpeza hospitalar: contribuições da ergonomia e da psicodinâmica do trabalho. Produção, São Paulo, v. 14, n. 3, p. 45-57, set./dez. 2004.

TAVARES, J. C. Análise do trabalho em agentes de contato-paciente: investigação das táticas utilizadas. Comportamento Organizacional e Gestão, Lisboa, v. 12, n. 2, p. 173-185, 2006.

VEIGA, A. R. Condições de trabalho, fatores de risco e problemas de saúde percebidos pelo trabalhador de enfermagem hospitalar. 2007. $120 \mathrm{f}$. Dissertação (Mestrado em Enfermagem)-Faculdade de Enfermagem, Universidade do Estado do Rio de Janeiro, Rio de Janeiro, 2007.

VOGEL, L. La evaluación de los riesgos en los centros de trabajo y la participación de los trabajadores. Cuadernos de Relaciones Laborales, Madrid, n. 7, p. 13-44, 1995.

ZAMBERLAN, C.; SIQUEIRA, H. C. H. A terceirização nos serviços e as conseqüências no cuidar em enfermagem. Revista Brasileira de Enfermagem, Rio de Janeiro, v. 58, n. 6, p. 727-730, nov./dez. 2005. 